\title{
The Emerging Web of Social Machines
}

\author{
Silvio R. L. Meira ${ }^{1,3}$, Vanilson A. A. Burégio ${ }^{1}$, \\ Leandro M. Nascimento ${ }^{1}$, Elaine Figueiredo ${ }^{1}$ \\ ${ }^{1}$ Informatics Center - Federal University of \\ Pernambuco - UFPE \\ [srlm,vaab,lmn,egmf]@cin.ufpe.br
}

\author{
Misael Neto ${ }^{2,3}$, Bruno Encarnação ${ }^{1}$, Vinícius \\ Cardoso Garcia ${ }^{1}$ \\ ${ }^{2}$ Catholic University of Pernambuco - UNICAP \\ ${ }^{3}$ C.E.S.A.R, Recife, PE, Brazil \\ misael.neto@cesar.org.br, [bpe,vcg]@cin.ufpe.br
}

\begin{abstract}
We define a notion of social machine and envisage an algebra that can describe networks of such. To start with, social machines are defined as tuples of input, output, processes, constraints, state, requests and responses; apart from defining the machines themselves, we define their connectors and conditionals that can be used to describe the interactions between any number of machines in a multitude of ways, as a means to represent real machines interacting in the real web, such as Twitter, Twitter running on top of Amazon AWS, mashups built using Twitter and, obviously, other social machines. This work is not a theoretical paper as yet; but, in more than one sense, we think we have found a way to describe web based information systems and are starting to work on what could be a practical way of dealing with the complexity of this emerging web of social machines that is all around us.
\end{abstract}

Keywords- social machines; web development; sociable application; programable web

\section{INTRODUCTION}

Nowadays, computing means connecting [1]; and it just may be the case that developing software is the same as connecting services [5]. Recently, we all can clearly see that a new phase is emerging, the web " 3.0 ", the web as a programming platform, the network as an infrastructure for innovation, on top of which all and sundry can start developing, deploying and providing information services using the computing, communication and control infrastructures in a way fairly similar to utilities such as electricity.

Although there have been many studies about the future of the internet and concepts such as web 3.0, programmable web [2], linked data [3] and semantic web [4], the segmentation of data and the issues regarding the communication among systems obfuscates the interpretation of this future. Unstructured data, unreliable parts and problematic, non-scalable protocols are all native characteristics of the internet that has been evolving for 40 years; at the same time, they are the good, the bad and the ugly of a web in which we rely more and more in the everyday life of everything, that needs a unifying view and explanations in order to be developed, deployed and used in a more efficient and effective way.

The read/write and programmable webs are recent enough to represent very serious difficulties in understanding their basic elements and how they can be efficiently combined to develop real, practical systems in either personal, social or enterprise contexts. There has not been a clear, precise description of each and every entity on this new emerging web and we believe it is necessary to create new mental models of such a web as a platform, in order to provide a common and coherent conceptual basis for the understanding of this young, upcoming and possibly highly innovative phase of software development.

\section{SOCIAL MACHINES: THE CONCEPT}

Motivated by these issues, we have tried to explain the web in terms of a new concept named Social Machines (SM). We define a SM as a tuple, as following: $S M=<R e l, W I$, Req, Resp, S, Const, I, P, O>

In general, a SM represents a connectable and programmable entity containing an internal processing unit $(P)$ and a wrapper interface (WI) that waits for requests (Req) from and replies [with responses (Resp)] to other social machines. Its processing unit receives inputs (I), produces outputs (O) and has states (S); and its connections define intermittent or permanent relationships (Rel) with other SMs, connections which are established under specific sets of constraints (Const). Figure 1 illustrates a basic representation of a Social Machine.

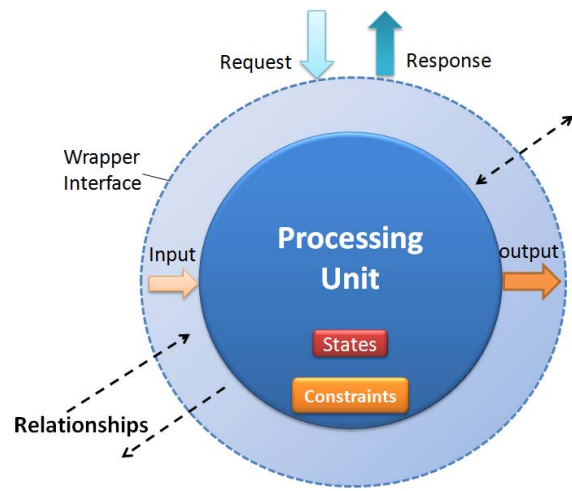

Figure 1 - A representation of a Social Machine

We are still doing the basic research on an algebra that can be used to describe these machines, their networks and interactions between any numbers of them; for now, let us briefly discuss about their main goal.

The idea behind Social Machines is to take advantage of the networked environment they are in there in to make it easier to combine and reuse exiting services from different SMs and use them to implement new ones. Hence, we can highlight some of its main characteristics, 
as following: Sociability, Compositionality, Platform and Implementation independency, Self-awareness, Discoverability and, but not least, Programmability.

By the very nature of the concept we are proposing, SMs are sociable stuff and, in nearly all cases, each one should provide means to interact with one another. The concept of relationships between SMs is similar to that of relationships between people; we can view them as trusted relations between different SMs, satisfying established constraints, which describe any restrictions that a given SM can have. Constraints can specify, for instance, authorization protocols (for security), number of requests per hour (for performance) and additional properties which can influence other quality attributes. This gives us the liberty to connect any number of SMs through the Web in order to form different networks and implement new services from the ones that already exist.

\section{CASE STUDY}

A real system (Futweet ${ }^{l}$ ) was developed using the unifying idea of social machines. Futweet is both a social network and a guessing game about football (soccer) results. Initially developed for Twitter users, Futweet was subsequently connected with other online social networks, e.g. Facebook and Orkut, making it a good case study for illustrating the development of an application that uses the concept of Social Machine. The game illustrates the development of a real social machine, since it was designed and built to be networked with other applications and be itself a connection point of other applications and services. The social game is an architecture of related machines (Twitter, Orkut, Facebook, Gmail and MSN) working on top of a virtual infrastructure (Amazon EC2). The functionalities of this network are encapsulated by a wrapper interface that makes the main features of the service available. It is important to note that the Futweet is the "glue code" between different social machines (with their own processing unit).

There are several factors that should be taken into account when developing a social machine; one has to bear in mind that the complexity of a given system's development is directly related to the properties, power, limitations and restrictions of other social machines considered in the project. Non-functional requirements can be affected by quality attributes of SMs being used as a basis for design and implementation, such as availability, limitations or restrictions of third party APIs, changes in the mechanisms for accessing social machines, among many other considerations.

We have give in that Futweet is a "toy" project when we compare to the usual scale of corporate projects; but, considering the functionality made available by the effort and comparing that to what would have been the effort of developing it from scratch, we are not talking about a small project anymore; glue it is, of course, but it is glue

\footnotetext{
${ }^{1}$ http://www.futweet.com.br
}

that keeps together quite a lot of stuff provided by other, existing, parts of -Social Machines in- the web. This may be quite a novel way to develop and evolve corporate information systems, and a somewhat radical departure from the two current competing efforts, the old "all from scratch" and the difficult "reusing reusable parts".

While developing Futweet we have learned a few practical things that are likely to be part of the process of construction of many other social machines; a nonexhaustive list ought to include: APIs access limits, error propagation, dynamic changes of machines over time, etc.

\section{CONCLUSION AND FUTURE DEVELOPNENTS}

The "emerging" in the title of this paper has a lot to do with that; for some time to come, it is likely that many people will resist designing applications in terms of SMs, especially when dependability is a serious matter, which is usually the case in (say) corporate systems.

However, in more than one sense, we think that this work can collaborate to the process of providing a unifying vision to describe web based information systems and are starting to work on what could be a practical way of dealing with the complexity of this emerging web. The impact of this change will be revolutionary. Starting to program social machines, each one will be able to create their own applications and provide new forms of articulation and expression in network.

For future developments we envisage a research framework containing a large number of research challenges which include (among many others): a language to specify SMs, an architectural framework for defining and developing SM-based Systems; and, but not least (and for completeness!), an evolution of the concept of Social Machines, considering people -peopleware and their inherently human capabilities- as an (old) new kind of Social (computing) Machine that can be used (like any other) in the development, deployment and evolution of applications on the emerging web of social machines.

\section{ACKNOWLEDGMENT}

This work was partially supported by the National Institute of Science and Technology for Software Engineering (INES, www.ines.org.br).

\section{REFERENCES}

[1] Roush, W. Social Machines - Computing means connecting. MIT Technology Review, August, 2005.

[2] Yu, S.; Woodard, C. Innovation in the programmable web: Characterizing the mashup ecosystem. Service-Oriented Computing--ICSOC 2008 Workshops, pp. 136-147, 2009.

[3] Christian Bizer, Tom Heath and Tim Berners-Lee: Linked Data The Story So Far. International Journal on Semantic Web and Information Systems, Vol. 5(3), 2009, Pages 1-22.

[4] P. Hitzler, M. Kroetzsch, S. Rudolph, A. McGettrick, and J. Impagliazzo, Foundations of Semantic Web Technologies, Chapman and Hall, 2009.

[5] Turner, M., Brereton, P., \& Budgen, D. (2003). Turning Software into a Service. Computer, 36(10), 38-44. 